\title{
The Clinical Efficacy of Low-Dose Tacrolimus Combined with Tripterygium to Treat the Steroid-Resistant Nephrotic Syndrome
}

\author{
Hongqi Ren ${ }^{1}$, Guofang Chen ${ }^{2}$, Xuan Zhou ${ }^{1}$, Yan Li ${ }^{1}$, Qing Cai ${ }^{1}$, Shujing Han ${ }^{1}$, Rui Wang ${ }^{1}$ \\ ${ }^{1}$ Department of Nephrology, Huaihai Hospital of Xuzhou Medical College, Xuzhou, China \\ ${ }^{2}$ Department of Neurology, Central Hospital of Xuzhou, Xuzhou, China \\ Email: sznk2005@yahoo.com.cn
}

Received September 30, 2012; revised November 2, 2012; accepted November 15, 2012

\begin{abstract}
Objective: To observe the clinical efficacy and safety of low dose tacrolimus (TAC) combined with tripterygium (TW) in treatment of steroid resistant nephritic syndrome (SRNS). Method: The patients, who were diagnosed with mesangial proliferative glomerulonephritis (MesPGN) and focal segmental glomerulosclerosis (FSGS) by biopsy and failed to respond to a 3-month treatment with prednisone $(1 \mathrm{mg} / \mathrm{kg} \cdot \mathrm{d})$, were randomly divided into 2 groups (TAC + TW Group and TW Group). Initially TAC + TW group took TAC $0.05 \mathrm{mg} /(\mathrm{kg} \cdot \mathrm{d}) 2 \mathrm{~h}$ after meal at $12 \mathrm{~h}$ interval. The plasma TAC level was examined after 3 days and was kept at $1.5-4 \mathrm{ng} \cdot \mathrm{ml}$; meanwhile, TW was given at $60 \mathrm{mg} / \mathrm{d}$ before meal. TW group only took TW $(60 \mathrm{mg} / \mathrm{d})$. The efficacy, adverse reactions and plasma TAC levels were observed in each group. Results: 1) Totally 20 SRNS patients completed the trial, 11 of TAC + TW Group and 9 of TW Group. There is no statistical difference between the two groups in terms of age, gender, duration since onset of the disease, blood pressure, $24 \mathrm{~h}$ UPQ, serum albumin, creatinine, cholesterol, triglyceride, FBG, kidney pathological categories, time of taking prednisone etc.; 2) Urine protein started to decrease after 1 month treatment in both of TAC + TW and TW groups. By the 12th month of treatment, TAC + TW group showed 8 cases of complete remission $(72.7 \%), 2$ cases of partial remission (18.2\%) and 1 case of no improvement $(9.1 \%)$, while those of TW groups were $2(22.2 \%), 4(44.5 \%)$ and 3 (33.3\%), respectively; 3) With treatment, the TAC + TW Group patients' plasma protein was significantly higher than that of pretreatment stage and recovered to normal level after 6 month of treatment. However, there was no significant plasma protein increase in TW Group. No obvious changes were observed on serum creatinine level of patients of both the two groups; 4) The incidence of adverse reactions was not significantly different between the two groups. Conclusion: TAC + TW reduced proteinuria of SRNS patients, increased clinical remission rate and was tolerant to SRNS patients. We conclude that TAC + TW treatment is an effective way to treat patients with SRNS.
\end{abstract}

Keywords: Steroid-Resistant Nephrotic Syndrome; Tacrolimus; Tripterygium; Treatment Outcome

\section{Introduction}

As an immune disease, primary nephrotic syndrome is usually treated with adrenocortical hormone and cytotoxic drugs in clinic. For patients whose clinical symptoms are not remitted completely or partially after being treated with hormone of standard dosage $(1 \mathrm{mg} / \mathrm{kg} / \mathrm{d})$ for at least 3 months, they are diagnosed with steroid-resistant nephrotic syndrome (SRNS) [1]. SRNS is usually presented, pathologically, as minimal change disease (MCD), mesangial proliferative glomerulonephritis (MesPGN) and focal segmental glomerulosclerosis (FSGS), with a few of membranous nephropathy and individual of non-IgA mesangial proliferative glomerulonephritis, which is difficult to be treated and generally immunosuppressive agents such as cyclophosphamide and cyclosporine
A are needed for the treatment in clinic [2,3].

Tacrolimus (TAC) is a new immunosuppressive agent, by suppressing calmodulin, the activation of T-cell, hyperplasia of T-helper-dependent B cell and the expression of lymphocyte factor and receptor to achieve immunodepression [1,2,4]. In 1990, Mccauley [5] reported children HRNS cases caused by the treatment of FSGS with TAC for the first time. Up to now, most of the treatment on primary nephrotic syndrome with TAC are still about the report of cases and only a few are about retrospective study and prospective study. And the treatment objects are hormone resistance and (or) dependent nephrotic syndromes patients mostly attacked FSGS and MCD [6,7]. In the 1980s, Leishi Li first used common threewingnut root, a traditional medicine on the treat- 
ment of renal glomerulus diseases and in the later sever aldecades, through clinical application, it has been found that tripterygium (TW) presents its unique curative effects in the treatment of many renal glomerulus diseases such as minimal change disease, IgM nephropathy and IgA nephropathy. Researches showed that common threewingnut root has obvious functions of anti-inflammation and immunosuppression; In addition, a series of basic researches in recent years have proved that common threewingnut root has unique functions of podocyte protection and it can reduce the urine protein amount of glomerulonephritis patients $[8,9]$.

Currently, certain curative effects have been achieved through the treatment of SRNS with sufficient and longtreatment TAC and double-dosage TW in clinic, but TAC is expensive and has certain side effects, so it is difficult to promote it in clinic. On the other hand double-dose TW has serious side effects such as liver dysfunction and paramecia for long-term use. The disease may easily recur if TW is reduced to single dose.

Therefore, it is not feasible for TW to be used in clinic for a long time. Based on the previous researches, we observed the effectiveness and safety of treatment of SRNS with small-dose TAC combined with single-dose TW, aiming to explore a cost-affordable method to treat SRNS.

\section{Target and Method}

\subsection{Case Selection}

20 patients hospitalized in our hospital from January 2007 to September 2009 are selected and all of the cases meet the following conditions: 1) Age: 18 - 85, gender: unlimited; 2) Clinically being diagnosed as glomerular mesangial hyperplasia cases and glomerulus FSGS cases through kidney biopsy; 3) Clinical manifestation: brine protein ration $>3.5 \mathrm{~g} / \mathrm{d}$, seralbumin $<30 \mathrm{~g} / \mathrm{L}$ and serum creatinine $(\mathrm{SCr})<132.6 \mu \mathrm{mol} / \mathrm{L}$; 4) No curative effects are observed on the patients after their taking metacortandracin $(1 \mathrm{mg} / \mathrm{kg} / \mathrm{d}$ and maximum $60 \mathrm{mg} / \mathrm{d})$ for above three months. The following situations are excluded: 1) Clear secondary cases such as systemic lupus erythematosus, hepatitis $\mathrm{B}$, hepatitis $\mathrm{C}$ virus infection, thyroid gland and tumor in other parts and poisoning of organic solvent and heavy metal; 2) Patients with serious complications, such as infection, cardiac insufficiency, liver dysfunction and disorders of galactose metabolism; 3) Patients who have received the treatment of cyclophosphamide (CTX), cyclosporin A (CsA), leflunomide and mycophenolate mofetil. This research has been approved by the Ethics Committee of our hospital and all patients have signed the Informed Consent before being arranged in the groups.

\subsection{Research Design and Treatment Plan}

\subsubsection{Random Grouping}

Before participating in the research, patients were applied to reduced dosage of metacortandracin $(20 \mathrm{mg} / \mathrm{d}$, reducing 5 - $10 \mathrm{mg}$ each week). Those patients were divided into TAC + TW Group and a TW Group randomly, with the group number of 11 and 9 respectively. The follow-up visit observation time is 12 months.

\subsubsection{Treatment Plans}

TAC + TW group: TAC produced by Astellas China Pharmaceutical Co., Ltd. (0.5 mg/pill and $1 \mathrm{mg} /$ pill $)$ is applied in the test. Patients took TAC $0.05 \mathrm{mg} / \mathrm{kg} / \mathrm{d} 2$ hours after meal every 12 hours. The whole blood TAC concentration was detected (by ELISA method, PROTrac II kit produced by DiaSorin Corporation) 3 days later. Later, TAC concentration was examined every month and the blood concentration was maintained at $1.5-4 \mathrm{ng} / \mathrm{ml}$. During the period of TAC treatment, $60 \mathrm{mg} / \mathrm{d}$ TW (produced by Taizhou Pharmaceutical Factory, $10 \mathrm{mg} /$ pill and 100 pills/bottle) were taken by patients be fore 3 meals. TW group: only TW was taken by patients before 3 meals, patients whose conditions are completely or partially remitted are required to continue taking it up to 12 months.

Reduction of metacortandracin: for TAC + TW Group, metacortandracin was reduced by $5 \mathrm{mg} / \mathrm{d}$ each week. Later, it was reduced by $5 \mathrm{mg}$ every other day each week when it was reduced to $20 \mathrm{mg} / \mathrm{d}$ and $2.5 \mathrm{mg} / \mathrm{d}$ every two weeks when reduced to $20 \mathrm{mg}$ every other day; and reduced gradually till after it was stopped. For TW group, metacortandracin was reduced by $5 \mathrm{mg}$ every two weeks; changed as reduction by $5 \mathrm{~m}$ every other day when it is reduced to $20 \mathrm{mg} / \mathrm{d}$ and $2.5 \mathrm{mg}$ every two weeks when reduced to $20 \mathrm{mg}$ every other day; and gradually reduced till it is stopped.

If liver dysfunction (alanine or glutamic-oxalacetic transaminease exceeds twice of the normal value) occurs or peripheral blood leukocyte reduces to $<3 \times 10^{9} / \mathrm{L}$ after medicine taking, TAC would be suspended and TW was taken continuously for a week in the original dosage; if the situation mentioned above can return to normal, TAC and TW can be taken continuingly in treatment dosage. If a patient shows serious complicating infection, the medicine shall be suspended and then be used continuingly after the infection is controlled for 2 weeks and the baseline standard shall be re-evaluated. In the treatment, patients stopping taking specified medicine for 2 weeks for various reasons shall not be considered anymore.

Other treatment: low-salt diet $(<3 \mathrm{~g} / \mathrm{d}$ sodium salt intake). If a patient has high-blood pressure, calcium antagonist was preferrably used to control the blood pressure to $120 / 80 \mathrm{mmHg}$; if ACEI and ARB have been 
adopted before treatment, they can be used continuingly; diuretic can be used for patients with obvious edema and statin lipid-lowing drugs can be used for patients with serious hyperlipidemia.

\subsection{Follow-Up Observation and Observation Indicators}

Follow-up observation: Follow-up observation must be carried out in the 2 nd week, and the 1 st, 3rd, 6th and 12th month after the start of the treatment. To observe patient's conditions, adverse reactions and laboratory examination were performed.

Laboratory examination: The $24 \mathrm{~h}$ urine protein quantitation was examined at the base line and each follow-up visit, and average value at each follow-up visit was calculated. In the 3 days before taking $24 \mathrm{~h}$ urine, patients were subject to regular diet, and were not allowed to take diuretic or transfuse albumin.

Efficacy observation: the urine protein level was observed at the 2 nd week, and the 1st, 3rd, 6th and 12th month after the beginning of treatment, and the values of plasma protein, liver function, serum creatinine, blood sugar and blood fat were recorded.

Adverse events: All adverse events in therapeutic process should be recorded in details, such as anaphylactic reaction, infection, gastrointestinal reaction, renal function impairment, liver function impairment, blood system impairment and female menses condition, etc.; if the values of glutamic-pyruvic transaminase or glutamic-oxaloacetic transaminase are twice of the upper limit of normal value, it can be defined as elevation of transaminase.

\subsection{Assessment of Efficacy $[9,10]$}

Complete remission $(\mathrm{CR})$ : urine protein concentration $<$ $0.4 \mathrm{~g} / 24 \mathrm{~h}$, serum albumin $>35 \mathrm{~g} / \mathrm{L}$, and stable renal function.

Partial remission (PR): urine protein level decreases and exceeds $50 \%$ of the base value, $\leq 3.5 \mathrm{~g} / \mathrm{d}$ and stable renal function.

No remission (NR): urine protein concentration is lower than $50 \%$ of the base value, or concentration $>3.5 \mathrm{~g} / \mathrm{d}$, or serum creatinine increases and exceeds $50 \%$.

Effective rate $=\mathrm{CR}$ rate $+\mathrm{PR}$ rate.

\section{Statistical Methods}

The inspection data were processed with SPSS 13.0 statistical software, and quantitative data were indicated by mean \pm standard deviation. Comparison among groups adopts $\mathrm{t}$ inspection. Enumeration data were indicated in the number of cases (percentage); comparison among groups adopts $\mathrm{X}^{2}$ for inspection, in which $\mathrm{P}<0.05$ represents that the difference has remarkable statistical significance and $\mathrm{P}<0.01$ represents extraordinary statistical significance.

\section{Results}

\subsection{General Conditions}

20 SRNS patients were selected, with 9 to TW Group and 11 to TAC + TW Group. There were no obvious statistical difference between the two groups in age, gender, duration since onset of the disease, blood pressure, 24 h UPQ, serum albumin, creatinine, cholesterol, triglyceride, FBG, kidney pathological categories, time of taking prednisone etc. (Table 1).

Table 1. Clinical characteristics (base line) comparison.

\begin{tabular}{|c|c|c|c|}
\hline & TW & $\mathrm{TAC}+\mathrm{TW}$ & $\mathrm{P}$ \\
\hline No. Patient & 9 & 11 & \\
\hline Age $(y r)$ & $40.7 \pm 11.9$ & $42.8 \pm 13.5$ & 0.36 \\
\hline Gender $(\mathrm{M} / \mathrm{F})$ & $6 / 3$ & $7 / 4$ & 0.90 \\
\hline Duration since onset of the disease (Mon) & $5.2 \pm 2.9$ & $4.0 \pm 2.2$ & 0.11 \\
\hline $\mathrm{BP}(\mathrm{mmHg})$ & $126.0 \pm 15.3$ & $125.3 \pm 15.7$ & 0.46 \\
\hline DBP (mmHg) & $75.1 \pm 11.8$ & $77.7 \pm 9.7$ & 0.29 \\
\hline Urine protein $(\mathrm{g} / 24 \mathrm{hr})$ & $5.6 \pm 1.9$ & $6.0 \pm 1.4$ & 0.28 \\
\hline $\mathrm{SCr}(\mathrm{umol} / 1)$ & $87.8 \pm 9.9$ & $89.1 \pm 9.6$ & 0.39 \\
\hline $\operatorname{Alb}(g / 1)$ & $24.3 \pm 2.6$ & $23.0 \pm 3.3$ & 0.18 \\
\hline Cho $(\mathrm{mmol} / \mathrm{l})$ & $8.9 \pm 1.4$ & $8.4 \pm 1.6$ & 0.23 \\
\hline $\operatorname{Tg}(\mathrm{mmol} / \mathrm{l})$ & $3.4 \pm 0.9$ & $3.0 \pm 0.6$ & 0.18 \\
\hline Histology & & & 0.88 \\
\hline MesPGN & 3 & 4 & \\
\hline FSGS & 6 & 7 & \\
\hline Duration of $1 \mathrm{mg} / \mathrm{kg} / \mathrm{d}$ prednisone (weeks) & $8.3 \pm 0.8$ & $9.2 \pm 2.0$ & 0.11 \\
\hline
\end{tabular}




\subsection{Clinical Efficacy}

For TAC + TW Group, 1 month after the treatment, the urine protein of patients started to decrease, with 0 case of complete remission, 3 cases of partial remission (27.3\%), and the total effective rate (27.3\%); 3 months after treatment: 2 cases of complete remission (18.2\%), 7 cases of partial remission (63.6\%), 2 cases of ineffectiveness (18.2\%), and the total effective rate was $81.8 \%$; 6 months after treatment: 3 cases of complete remission (27.3\%), 7 cases of partial remission (63.5\%), 1 case of ineffectiveness $(9.1 \%)$ and the total effective rate was $90.9 \%$; when the follow-up visits were carried out till the 12th month: 8 cases of complete remission $(72.7 \%), 2$ cases of partial remission (18.2\%), 1 case of ineffectiveness $(9.1 \%)$ and the total effective rate was $90.9 \%$. When the follow-up visits were over, the complete remission rate of TAC + TW Group was higher than that of TW Group (Table 2).

For TW Group, 1 month after the treatment, urine protein of patients also started to decrease, with 0 case of complete remission, 2 cases of partial remission; 3 months after treatment: 0 case of complete remission, 5 cases of partial remission (55.6\%), 4 cases of ineffectiveness (44.4\%), and the total effective rate was $55.6 \%$; 6 months after treatment: 1 case of complete remission (11.1\%), 5 cases of partial remission (55.6\%), 3 cases of ineffectiveness $(33.3 \%)$, and the total effective rate was $66.7 \%$; when the follow-up visits were carried out till the 12th month: 2 cases of complete remission (22.2\%), 4 cases of partial remission (44.5\%), 3 cases of ineffectiveness (33.3\%), and the total effective rate was $66.7 \%$.

\subsection{Changes of Observation Indicators of the Two Groups before and after Treatment}

For TAC + TW Group, comparing with the base line values, the urine protein values of patients have decreased obviously to $28.3 \% 、 69.5 \% 、 82.3 \%$ and $86.2 \%$ at the 1 st, $3 \mathrm{rd}, 6$ th and 12th month after the beginning of treatment, which are obviously higher than those of TW Group (Table 4, Figure 1 (a)). For TW Group, the urine protein values of patients have also decreased to $28.3 \%, 44.7 \%$, $52.4 \%$ and $59.2 \%$ at the $1 \mathrm{st}, 3 \mathrm{rd}$, 6th and 12 th month after the beginning of treatment. The conclusion is: for TW Group, the urine protein value decrease examined one month after treatment has statistical significance; the decrease examined at the end of the test, although still has statistical significance comparing with the base line values, is at a low level of only $59.2 \%$ (Table 3 ).

Table 2. Efficacy comparison of follow-up visits until the end of the trial.

\begin{tabular}{ccccc}
\hline & PR & CR & NR & $\begin{array}{c}\text { Total effective } \\
\text { rate }\end{array}$ \\
\hline TW & $2(22.2 \%)$ & $4(44.5 \%)$ & $2(33.3 \%)$ & $66.7 \%$ \\
TAC + TW & $8(72.7 \%)$ & $2(18.2 \%)$ & $1(9.1 \%)$ & $90.9 \%$ \\
\hline
\end{tabular}

The complete remission rate of TAC+TW group is higher than that of TW group when follow-up visits are ended, $\mathrm{P}=0.024<0.05$.

Table 3. Changes of urine protein and biochemical indicators of TW group before and after treatment.

\begin{tabular}{|c|c|c|c|c|c|c|}
\hline & \multirow{2}{*}{ Pre-treatment } & \multicolumn{5}{|c|}{ Post-treatment } \\
\hline & & $2 w$ & $1 \mathrm{~m}$ & $3 \mathrm{~m}$ & $6 \mathrm{~m}$ & $12 \mathrm{~m}$ \\
\hline Urine protein $(\mathrm{g} / 24 \mathrm{hr})$ & $5.6 \pm 1.9$ & $5.2 \pm 1.0$ & $4.4 \pm 0.9^{\mathbf{\Lambda}}$ & $3.1 \pm 1.4^{\mathbf{\Lambda \Lambda}}$ & $2.7 \pm 1.3^{\mathbf{\Lambda \Lambda}}$ & $2.3 \pm 1.2^{\mathbf{\Lambda}}$ \\
\hline $\mathrm{SCr}(\mathrm{umol} / \mathrm{l})$ & $87.8 \pm 9.9$ & $86.5 \pm 6.9$ & $83.2 \pm 10.6$ & $80.3 \pm 12.2$ & $84.1 \pm 5.8$ & $79.9 \pm 9.2$ \\
\hline Alb (g/l) & $24.3 \pm 2.6$ & $25.5 \pm 2.3$ & $26.2 \pm 2.4^{\mathbf{\Lambda}}$ & $27.8 \pm 1.8^{\mathbf{\Lambda}}$ & $29.3 \pm 1.9^{\boldsymbol{\Lambda} \Lambda}$ & $30.9 \pm 2.1^{\Delta \wedge}$ \\
\hline Cho (mmol/l) & $8.9 \pm 1.4$ & $8.3 \pm 1.5$ & $7.4 \pm 1.0^{\mathbf{\Lambda}}$ & $5.5 \pm 2.1^{\wedge}$ & $4.4 \pm 1.4^{\mathbf{\Lambda \Lambda}}$ & $5.0 \pm 1.7^{\Lambda \Lambda}$ \\
\hline $\operatorname{Tg}(\mathrm{mmol} / \mathrm{l})$ & $3.4 \pm 0.9$ & $2.9 \pm 0.6$ & $2.9 \pm 0.7$ & $2.2 \pm 0.5^{\mathbf{\Lambda}}$ & $2.3 \pm 0.5^{\boldsymbol{\Lambda}}$ & $2.2 \pm 0.6^{\mathbf{\Lambda}}$ \\
\hline GLU (mmol/l) & $5.7 \pm 0.7$ & $5.3 \pm 0.5$ & $5.4 \pm 0.5$ & $5.5 \pm 0.7$ & $5.1 \pm 0.8$ & $5.2 \pm 0.4$ \\
\hline
\end{tabular}

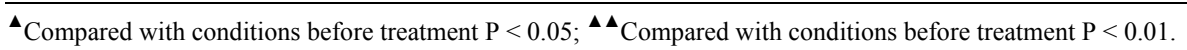

Table 4. Changes of urine protein and biochemical indicators of TAC + TW group before and after treatment.

\begin{tabular}{|c|c|c|c|c|c|c|}
\hline & \multirow{2}{*}{ Pre-treatment } & \multicolumn{5}{|c|}{ Post-treatment } \\
\hline & & $2 \mathrm{w}$ & $1 \mathrm{~m}$ & $3 \mathrm{~m}$ & $6 \mathrm{~m}$ & $12 \mathrm{~m}$ \\
\hline Urine protein $(\mathrm{g} / 24 \mathrm{hr})$ & $6.0 \pm 1.4$ & $5.1 \pm 1.4$ & $4.3 \pm 1.3^{\boldsymbol{\Lambda} \boldsymbol{\Lambda}}$ & $1.8 \pm 1.4^{\mathbf{\Lambda \Lambda}}$ & $1.1 \pm 1.0^{\mathbf{\Lambda}}$ & $0.9 \pm 0.9^{\boldsymbol{\Lambda} \Lambda}$ \\
\hline $\mathrm{SCr}$ (umol/l) & $89.1 \pm 9.6$ & $83.5 \pm 6.6$ & $82.9 \pm 10.4$ & $84.4 \pm 5.1$ & $81.3 \pm 12.2$ & $81.6 \pm 10.8$ \\
\hline Alb (g/l) & $23.0 \pm 3.3$ & $24.1 \pm 2.4$ & $29.8 \pm 2.3^{\boldsymbol{\Lambda}}$ & $31.5 \pm 5.5^{\mathbf{\Lambda}}$ & $37.4 \pm 1.8^{\mathbf{\Lambda}}$ & $37.1 \pm 1.3^{\mathbf{\Lambda}}$ \\
\hline Cho (mmol/l) & $8.4 \pm 1.6$ & $8.0 \pm 1.1$ & $6.6 \pm 1.2^{\boldsymbol{\Lambda} \Delta}$ & $4.9 \pm 1.1^{\mathbf{\Lambda}}$ & $5.4 \pm 1.9^{\mathbf{\Lambda} \mathbf{\Lambda}}$ & $4.8 \pm 1.1^{\mathbf{\Lambda} \Lambda}$ \\
\hline $\operatorname{Tg}(\mathrm{mmol} / \mathrm{l})$ & $3.0 \pm 0.6$ & $2.8 \pm 0.5$ & $2.8 \pm 0.5$ & $2.3 \pm 0.4^{\mathbf{\Lambda}}$ & $2.2 \pm 0.6^{\mathbf{\Lambda}}$ & $1.9 \pm 0.4^{\mathbf{\Lambda}}$ \\
\hline GLU (mmol/l) & $5.8 \pm 0.4$ & $5.4 \pm 0.8$ & $5.4 \pm 0.6$ & $5.6 \pm 0.6$ & $5.3 \pm 0.7$ & $5.7 \pm 0.4$ \\
\hline
\end{tabular}

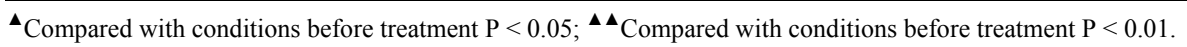


After treatment, plasma protein of TAC + TW Group increased obviously; when treatment was carried out for 6 months, plasma protein basically recovered to the normal level (Table 4, Figure 1(a)); however, while urine protein of TW Group decreased, although plasma albumin increased, the increase was obviously lower than that of TAC + TW group (Figure 1(b)). The serum creatinine level of patients in two groups had no obvious change before and after treatment (Tables $\mathbf{3}$ and 4, and Figure 1(c)). In the follow-up visits of TAC + TW group, no patient's serum creatinine has exceeded $50 \%$ of the basic value. The cholesterol and triglyceride values of patients in two groups decreased obviously compared with those values before treatment (Tables 3 and 4), but the decline range of cholesterol of patients in two groups were basically similar with no obvious statistical difference (Figure 1(d)). The fasting blood-glucose level of patients in two groups had no obvious change.

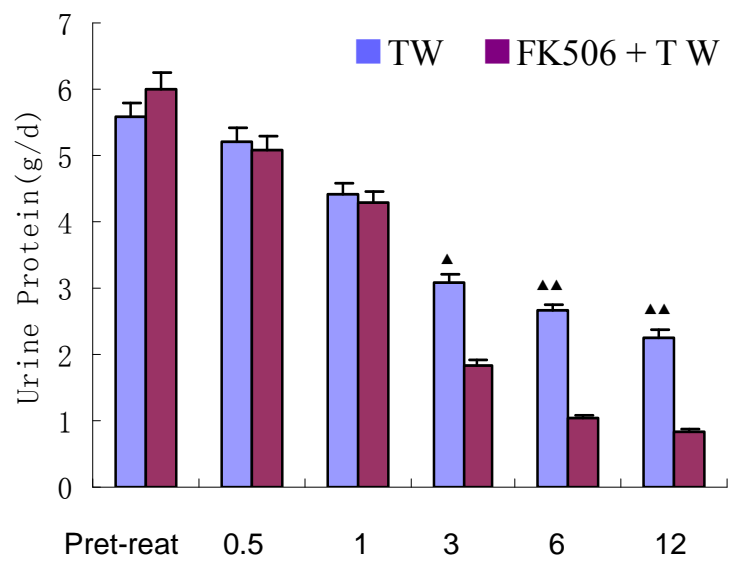

(a)

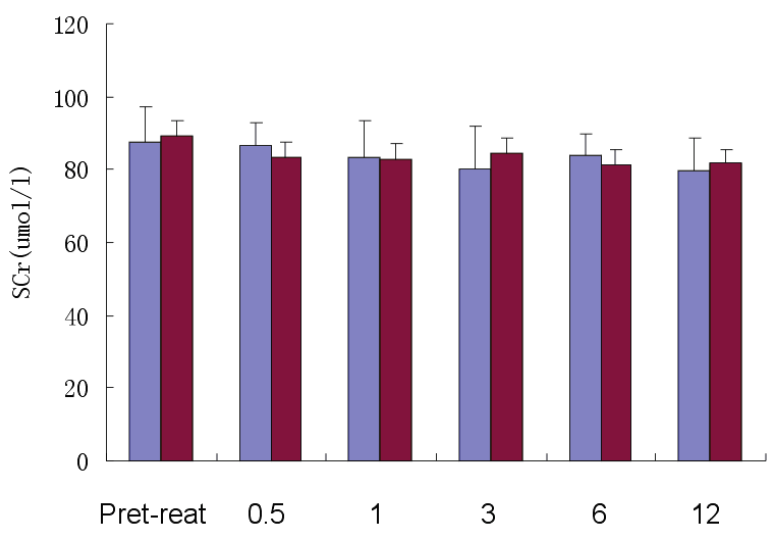

(c)

\subsection{TAC Concentration Change}

The TAC blood concentration of TAC + TW group was maintained at $1.5-3 \mathrm{ng} / \mathrm{ml}$, and the average blood concentration was relatively stable during treatment, but 6 months after treatment, the TAC content of patients of complete remission was reduced to $0.5 \mathrm{mg} 2$ /day (Figure 2).

\subsection{Adverse Reactions}

Generally, adverse reactions are not severe and will maintain for a short period of time. 2 cases of nausea and 1 case of herpes zoster happened to TAC + TW group, and 1 month after treatment, there were 3 cases with slightly increase of glutamic-pyruvic transaminase, and menstrual disorder happened to 2 cases of 4 female cases; no increase of serum creatinine exceeding $50 \%$ of base line value, and the fasting blood-glucose had no ob-

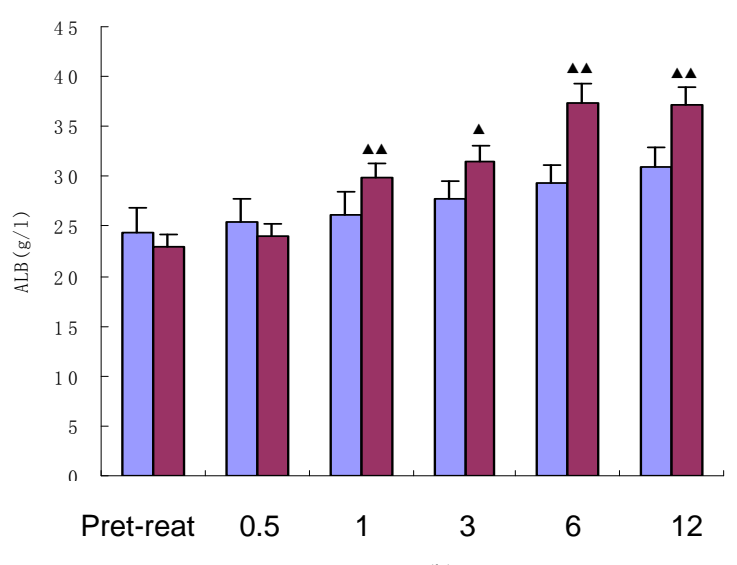

(b)

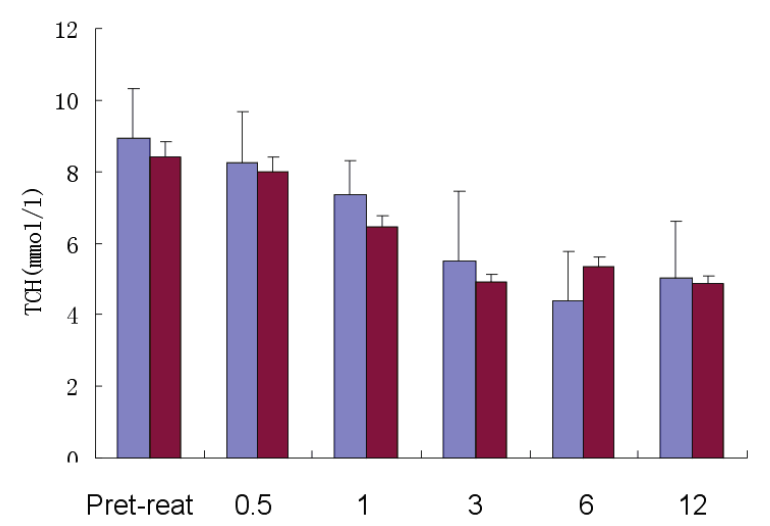

(d)

Figures 1. Change of urine protein and plasma albumin of TAC + TW group and TW group before and after treatment. (a) Follow-up consultation and comparison of urine protein of TAC + TW group and TW group; (b) Follow-up consultation and comparison of plasma albumin of TAC + TW group and TW group; (c) Follow-up consultation and comparison of serum creatinine of TAC + TW group and TW group; (d) Follow-up consultation and comparison of cholesterol of TAC+TW group and TW group. ${ }^{\wedge}$ Compared with conditions before treatment $P<0.05 ;{ }^{\wedge}$ Compared with conditions before treatment $P<$ 0.01. 


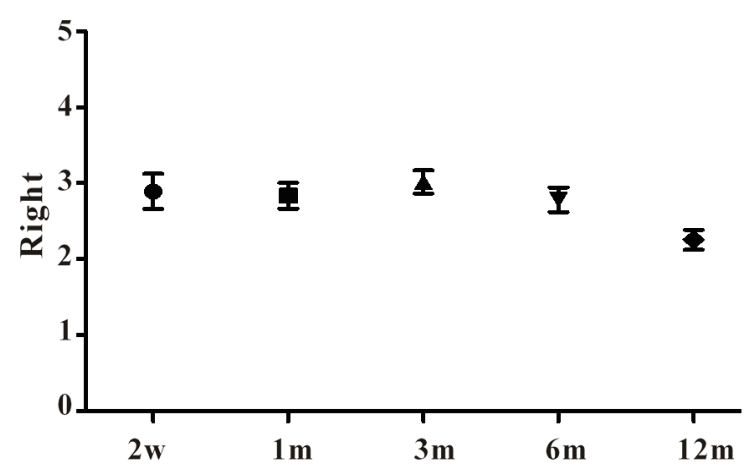

Figure 2. TAC Concentration Change of TAC+TW group.

vious increase. 1 case of nausea and 1 case of laxativeness happened to TW group, and after treatment of 1 month, there were 2 cases of slightly increase of glutamic-pyruvic transaminase, and menstrual disorder happened to 1 case of 3 females. Patients with increase of transaminase in two groups were given bifendate for symptomatic treatment and patients' conditions were improved; all patients were kept taking medicines and the treatment as well as follow-up visits were not affected.

\section{Discussion}

Therapeutic methods available for SRNS include the application of cyclophosphamide, cyclosporine A, implosive therapy of high-dose methylprednisolone, ACEI or ARB and mycophenolate mofetil, etc. Most researches reported that the effective rate of SNRS treatment is about $50 \%$ - $60 \%$, and patients who have no response to treatment are apt to develop to end-stage renal failure [10].

TAC is a calmodulin inhibitor, which is mechanistically similar to CsA, and its inhibiting effect on cell factor is significantly stronger than that of CsA. In recent years, there were many reports about treating SRNS with TAC. Gulati et al. [11] treated 19 SRNS patients with the combination of TAC and hormone. When the treatment was carried out for 3 months, 16 patients were completely remitted with a complete remission rate of $84 \% ; 2$ cases partial remission with a partial remission rate of $10.5 \%$. Li and other researchers (references needed here) treated SRNS patients with TAC combined with lowdose hormone, with the results indicating that the complete and partial remission rate were $64.7 \%$ and $17.6 \%$ after treatment of 24 weeks in TAC group. However, the TAC concentrations were relatively high, which fluctuated at $9.54 \pm 5.13 \mathrm{ng} / \mathrm{ml}$, and SRNS is prone to recur if the dose is reduced.

TW plays a unique role in the treatment of many inflammations, autoimmune diseases and organ transplantation rejections. In recent years, in vitro experiments have indicated that triptolide can stabilize the skeleton structure of podocyte and antagonize the damage of puromycin aminonucleoside (PAN) to podocyte, and when podocyte is damaged, triptolide can reverse the damage of podocytes and recover the skeleton structure of podocytes and the expression of relevant molecules [8]. Currently, the influence of triptolide on podocytes is considered to be an important factor for the decrease of urine protein of patients with glomeru-lonephritis [8,9]. With the research on the influence of glomerular podocytes on proteinuria production, people have recognized that for some diseases of the renal glomeruli which are characterized in a great quantity of proteinuria and accompanied by the damage to podocyte such as minute lesion, focal segmental glomerulo-sclerosis and membranous nephropathy, TW works well on reducing proteinruia to all the above diseases $[9,12]$.

However, the problems exist: hormonotherapy which is necessarily to be used in combination with TAC and TW in the treatment of SRNS always cause acne, central obesity and other side effects because of the high dose of hormone and long period of application [13-17]; In addition, high-dose TAC and double-dose TW also can cause moreside effects [4,9]. Therefore, first of all, among domestic institutions, we studied the feasibility of the treatment of SRNS with low-dose TAC (blood concentration 1.5 - $4 \mathrm{ng} / \mathrm{ml}$ ) combined with single-dose TW.

The research results are: 3 months after treatment with low-dose TAC combined with TW, there were 2 cases of complete remission out of 11 cases $(18.2 \%)$, and 7 cases of partial remission $(63.6 \%)$; 6 months after treatment there were 3 cases of complete remission out of 11 cases (27.3\%), and 7 cases of partial remission (63.5\%); when the follow-up visits were carried out till the 12 th month, 8 cases were completely remitted out of 11 cases $(72.7 \%)$ and 2 cases were partially remitted $(18.2 \%)$. The complete remission rate was obviously higher than that of TW group. In addition, our research also discovered that after the treatment with TAC combined with TW, the plasma protein of SRNS patients increased obviously, and when treatment was carried out for 6 months, it basically recovered to the normal level; despite that urine protein of TW group decreased and plasma albumin increased, the increase range was significantly lower than that of TAC + TW group. Therefore, it is indicated that the treatment of SRNS with low-dose TAC combined with TW would reach the expected efficacy.

The main renal histopathological symptoms of patients with primary nephrotic syndrome are: minimal change disease (MCD), focal segmental glomerulosclerosis (FSGS), membranous nephropathy (MN), MSPGM and MPGN. Current researches have showed that MCD is caused by damage to podocytes which is caused by nephrotoxic cell factors mediated and generated by abnormal immune 
response of T cells; FSGS is not only caused by abnormal permeability of slit membrane of podocytes which is caused by genetic defect, the occurrence of some FSGS is similar to that of MCD, which is related to the abnormal immune response of T cells; and MesPGN is a pathological change involving many Immune factors [18-21]. Therefore, we applied TAC to reduce the generation of immune complex by inhibiting the abnormal immune response of T cells; meanwhile, TW was combined not only to strengthen TAC anti-inflammation and anti-immunization, but also to reverse the damage to podocytes and recover the skeleton structure of podocyte and the expression of relevant molecules, so as to realize better efficacy [18]. Moreover, it is a better choice for patients who have clinical side effects caused by hormone as well as those who are hormone-phobic.

For the obvious increase of plasma protein of SRNS patients who received the treatment with low-dose TAC combined with TW, the plasma protein basically recovered to the normal level when treatment was carried out for 6 months; but the plasma protein of TW group was not obviously increased. One potential mechanism may be that TAC can assist the liver to produce albumin; However, TW has inhibiting effect on the synthesis of albumin of liver, and the exact mechanism is to be explored $[4,12]$.

Common side effects of TAC include: gastrointestinal reactions, immunosuppression, infection, elevated blood pressure, anemia, renal toxicity, increased blood sugar, etc. [4,22]. Adverse reactions of TW mainly include nausea, vomit, anorexia, abdominal distention, reduction of leukocyte, liver dysfunction and influences on menstrual cycle of female patients of childbearing age [9,12,17]. In our research, side effects in low-dose TAC + TW group included 2 cases of nausea and 1 case of herpes zoster, 3 cases of slightly increase of glutamic-pyruvic transaminase after treatment of 1 month, and 2 cases menstrual disorder out of 4 female cases; there was no discovery of serum creatinine exceeding $50 \%$ of the base line, and the fasting blood-glucose did not increase obviously. Patients with increased transaminase were given bifendate for symptomatic treatment and patients' conditions improved, all patients kept taking medicines and the treatment and follow-up visits were not affected. Our research indicated that the treatment of SRNS with low-dose TAC combined with TW would not generate serious side effects.

In conclusion, low-dose TAC + TW group may effecttively reduce the proteinuria of SRNS patients, increase the level of plasma albumin, has obvious effective remission rate, does not obviously increase side effects, which indicate that low-dose TAC + TW may be applied to the treatment of SRNS. However, since this research is a single-center research, and the number.

\section{REFERENCES}

[1] M. van Husen and M. J. Kemper, "New Therapies in Steroid-Sensitive and Steroid-Resistant Idiopathic Nephrotic Syndrome," Pediatric Nephrology, Vol. 26, No. 6, 2011, pp. 881-892. doi:10.1007/s00467-010-1717-5

[2] X. F. Sun and X. M. Chen, "Clinical Application of Tacrolimus in Primary Nephrotic Syndrome," Chinese Journal of Practical Internal Medicine, Vol. 28, No. 5, 2008, pp. 323-325.

[3] H. M. Li and X. D. Shi, "Treatment of Steroid-Resistant Nephrotic Syndrome with Tacrolimus," The Journal of Practical Medicine, Vol. 25, No. 22, 2009, pp. 3871-3873.

[4] Q., Chen, Z. H. Liu, W. X. Hu, et al., “A Prospective Preliminary Study of Tacrolimus (FK506) Combined with Low-Dose Prednisone in Patients with Membranous Lupus Nephropathy," Chinese Journal of Nephrology, Dialysis \& Transplantation, Vol. 13, No. 2, 2004, pp. 101106.

[5] J. McCauley, R. Shapiro, V. Scantlebury, et al., "FK 506 in the Management of Transplant-Related Nephrotic Syndrome and Steroid-Resistant Nephrotic Syndrome," Transplantation Proceedings, Vol. 23, No. 6, 1991, pp. 33543356.

[6] S. Choudhry, A. Bagga, P. Hari, et al., "Efficacy and Safety of Tacrolimus versus Cyclosporine in Children with Steroid-Resistant Nephrotic Syndrome: A Randomized Controlled Trial," American Journal of Kidney Diseases, Vol. 53, No. 5, 2009, pp. 760-769. doi:10.1053/j.ajkd.2008.11.033

[7] X. Li, H. Li, H. Ye, et al., "Tacrolimus Therapy in Adults with Steroid- and Cyclophosphamide-Resistant Nephrotic Syndrome and Normal or Mildly Reduced GFR," American Journal of Kidney Diseases, Vol. 54, No. 1, 2009, pp. 51-58. doi:10.1053/j.ajkd.2009.02.018

[8] Z. H. Chen, W. S. Qin, C. H. Zeng, et al., "Triptolide Reduces Proteinuria in Experimental Membranous Nephropathy and Protects against C5b-9-Induced Podocyte Injury in Vitro," Kidney International, Vol. 77, No. 11, 2010, pp. 974-988. doi:10.1038/ki.2010.41

[9] Z. H. Liu, S. J. Li, Y. Wu, et al., "Treatment of Membranous Nephropathy with Tripterygium Combined LowDose Hormone: A Prospective Randomized Control Trial," Chinese Journal of Nephrology, Dialysis \& Transplantation, Vol. 18, No. 4, 2009, pp. 303-309.

[10] I. Roberti and S. Vyas, "Long-Term Outcome of Children with Steroid-Resistant Nephrotic Syndrome Treated with Tacrolimus," Pediatric Nephrology, Vol. 25, No. 6, 2010, pp. 1117-1124. doi:10.1007/s00467-010-1471-8

[11] S. Gulati, N. Prasad, R. K. Sharma, et al., "Tacrolimus: A New Therapy for Steroid-Resistant Nephrotic Syndrome in Children," Nephrology Dialysis Transplantation, Vol. 23, No. 3, 2008, pp. 910-913. doi:10.1093/ndt/gfm688

[12] Y. C. Ge, H. L. Xie, S. J. Li, et al., "Effect of Tripterygium in Patients with Diabetic Nephropathy: A Prospective Randomized Control Clinical Trial," Chinese Journal of Nephrology, Dialysis \& Transplantation, Vol. 19, No. 6, 2010, pp. 501-507.

[13] M. Chen, H. Li, X. Y. Li, et al., "Tacrolimus Combined 
with Corticosteroids in Treatment of Nephrotic Idiopathic Membranous Nephropathy: A Multicenter Randomized Controlled Trial," American Journal of the Medical Sciences, Vol. 339, No. 3, 2010, pp. 233-238. doi:10.1097/MAJ.0b013e3181ca3a7d

[14] T. Aizawa-Yashiro, K. Tsuruga, S. Watanabe, et al., "Novel Multidrug Therapy for Children with Cyclosporine-Resistant or -Intolerant Nephrotic Syndrome," Pediatric Nephrology, Vol. 26, No. 8, 2011, pp. 1255-1261. doi:10.1007/s00467-011-1876-Z

[15] S. Tang, A. W. Tang, M. K. Tam, et al., "Use of Tacrolimus in Steroid- and Cyclophosphamide-Resistant Minimal Change Nephrotic Syndrome," American Journal of Kidney Diseases, Vol. 42, No. 5, 2003, pp. E13-E15. doi:10.1016/j.ajkd.2003.07.011

[16] L. Butani and R. Ramsamooj, "Experience with Tacrolimus in Children with Steroid-Resistant Nephrotic Syndrome," Pediatric Nephrology, Vol. 24, No. 8, 2009, pp. 1517-1523. doi:10.1007/s00467-009-1220-z

[17] G. F. Yuan and S. G. Yuan, "Clinical Observation on Treatment of Refractory Nephrotic Syndrome with Tripterygium Combined Prednisone," Chinese Journal of Integrated Traditional and Western Nephrology, Vol. 10, No. 3, 2009, pp. 246-247.
[18] L. N. Miao, J. Sun, H. Yuan, et al., "Clinical Study of Low Dosage FK506 in the Treatment of Mesangial Proliferative Glomerulonephritis," Chinese Journal of Nephrology, Vol. 21, No. 10, 2005, pp. 624-625.

[19] T. H. Westhoff and M. van der Giet, "Tacrolimus in the Treatment of Idiopathic Nephrotic Syndrome," Expert Opinion on Investigational Drugs, Vol. 16, No. 7, 2007, pp. 1099-1110. doi:10.1517/13543784.16.7.1099

[20] M. Del Rio and F. Kaskel, "Evaluation and Management of Steroid-Unresponsive Nephrotic Syndrome," Current Opinion in Pediatrics, Vol. 20, No. 2, 2008, pp. 151-156. doi:10.1097/MOP.0b013e3282f4e6e4

[21] M. D. Sinha, R. MacLeod, E. Rigby, et al., "Treatment of Severe Steroid-Dependent Nephrotic Syndrome (SDNS) in Children with Tacrolimus," Nephrology Dialysis Transplantation, Vol. 21, No. 7, 2006, pp. 1848-1854. doi:10.1093/ndt/gfi274

[22] S. Manrique-Rodríguez, C. M. Fernandez-Llamazares and M. Sanjurjo-Saez, "Pharmacotherapeutic Review and Update of Idiopathic Nephrotic Syndrome in Children," Pharmacy World \& Science, Vol. 32, No. 3, 2010, pp. 314-321. doi:10.1007/s11096-010-9380-2 